DOE/ER/13447-5

\title{
Investigations and Calculations Toward Increasing the Efficiency of Muon Catalyzed Fusion
}

Final Report

May 1, 1989 - November 30, 1989

Principal Investigator: H.J. Monkhorst

Department of Physics

University of Florida

Gainesville, Florida 32611

November 1989

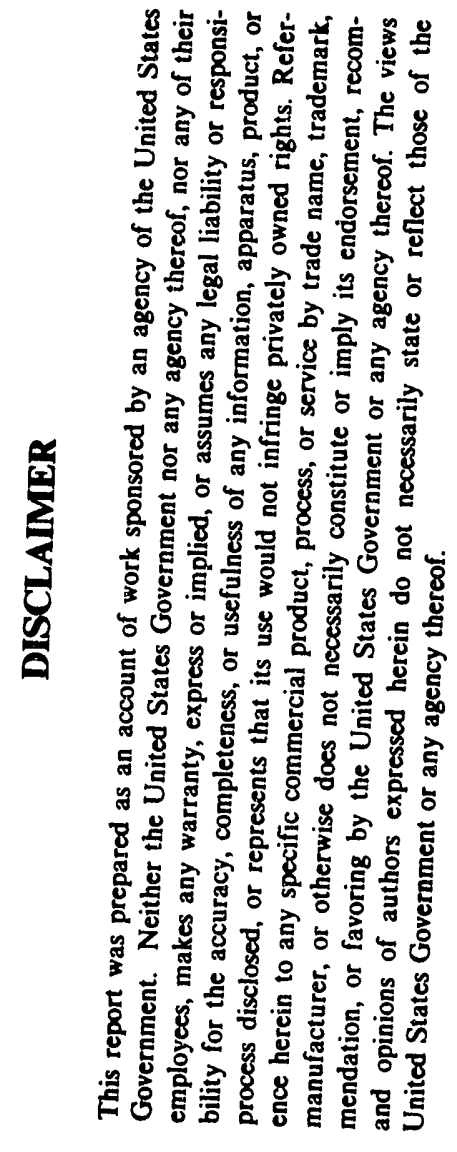

Prepared for the U.S. Department of Energy, Division of Advanced Energy Projects under grant number DE-FG05-85ER 13447. 
The previous progress report, dated January 1989 (DOE/ER/13447-4) summarized the work under the present grant between May 1, 1988 and April 30, 1989. Earlier reports, in aggregate, have covered the work under this grant from September 1985 forward. This, final report for this grant covers the activity period from May, 1989 till November 30, 1989. Publications prepared prior to this period have now all appeared in refereed journals, and the "Muon Catalyzed Fusion Workshop 1988," Proceedings in the AIP Series (Vol. 181). During this report's period eight papers were prepared, one of which has already appeared in print and the others are in various review and publication stages. Two additional papers are being prepared, bringing to a conclusion the work supported under this grant. Below I will summarize the results from this last period by category of completeness.

\section{A. Results in Preprint Form}

All results in this section are submitted for publication, and several are in the press. Some were covered in the "Results being Published" section of the previous progress report. As there have been changes since that report, it is necessary to revisit them.

Although several calculations for sticking fractions and d-t fusion from $\mathrm{dt} \mu(\mathrm{Jv})$ states had been published, the lingering discrepancy with experiment warranted another go at it, with and without inclusion of nuclear effects. In addition, $\mathrm{dd} \mu$ sticking fractions were obtained, supposedly in full agreement with experiment to three significant figures. In Ref. [1] we report sticking fractions for all the $\mathrm{J}=0$ and $\mathrm{J}=1$ states of $\mathrm{dt} \mu$ as well as $\mathrm{dd} \mu$, using random tempered basis sets. Very satisfactory convergence was observed, confirming earlier $\mathrm{dt} \mu(00)$ and $\mathrm{dt} \mu(01)$ results, but differing substantially for other states. In particular, the sticking for $\operatorname{dd} \mu(11)$, the formation and fusing state, disagrees with (is larger than) the experimental value by about $7 \%$. This is statistically significant, and raises also here the question about nuclear effects, or experimental problems.

In another report [2], also in the press, we addressed a more accurate calculation of the reactivation coefficient in d-t fusion. The error bar was lowered by a factor two, giving more confidence that the discrepancy with experiment does not arise from this phenomenon. A density dependence was found that is much weaker than that observed by the LAMPF experiments, and similar to that found at PSI. Within our error bars we agree with the calculations of Struensee and Cohen.

Fusion rates for all $\mathrm{xy} \mu(\mathrm{Jv})(\mathrm{Jv}=0,1)$ have been completed from our library of wavefunctions [3]. Intriguing discrepancies with other calculations, as well as some experiments for dd fusion are found. This is important because, again, it might point to nuclear effects, or a problem with the heuristic formula used.

In Ref. [4] we noted some errors made by Frolov et al in earlier muo-molecular calculations. We report the latest wavefunctions and energies better than any obtained before, and discuss the source of Frolov's errors.

In Ref. [5], together with many others at Delaware as well as Florida, we reported the work on nuclear effects on energy shifts and fusion rates for $(\mathrm{J}=0)$ states of $\mathrm{dt} \mu$, which was 
started while Dr. Szalewicz was still at UF. Substantial agreement, but also some systematic discrepancies with other results, were found. A logarithmic boundary condition consistent with a pure $\alpha-n$ outgoing wave was adopted within the R-matrix formalism.

Three contributions to the ongoing debate over the alleged phenomenon of cold (electroanalytic) fusion were completed. In Ref. [6] and [7], we addressed the d-d fusion rates in $D_{2}$ (or D-like) molecules. These were calculated both accurately, and with the WKB approximation. We showed that under believable conditions these rates are smaller by tens of orders of magnitude than experimentally claimed. A neat, and reliable closed formula was derived with WKB, and using the Born-Oppenheimer nature of the d-d dynamics description. In Ref. [8], we looked for possible fission/fusion chain reactions following source $d-d$ fusions. We concluded, again, that such reactions will not occur to a measurable extent, again leaving any heat generation in the experiments unexplained.

\section{B. Results being Prepared for Publication}

Two completed subprojects under this grant are now being prepared for publication. The major one, the complete, accurate calculation of relativistic, nuclear finite size and deuteron quadrupole moment corrections for $\mathrm{dt} \mu(11)$ and $\mathrm{dd} \mu(11)$ states have led to energies and hyperfine wavefunctions of very high quality. Energies were computed to better than $0.01 \mathrm{meV}$ accuracies. However, due to experimental uncertainties in the nuclear masses and electric + magnetic form factors the reliability is $\pm 0.5 \mathrm{meV}$. Preliminary results were included in a major review article by Cohen, Leon, Breunlich and Kammel. Comparison with other calculations by Bakalov and Korobov is favorable, but our data are better converged. The recommended relativistic + other corrections for these state are

$$
\begin{aligned}
& \Delta \mathrm{E}_{\text {corr }}[d t \mu(11)]=64.0 \pm 0.5 \mathrm{meV} \\
& \Delta \mathrm{E}_{\text {corr }}[d d \mu(11)]=24.6 \pm 0.5 \mathrm{meV}
\end{aligned}
$$

Energies and wavefunctions for all other systems $\mathrm{xy} \mu(\mathrm{Jv})$ and their hyperfine levels, will be published separately in tabular form. These quantities could be valuable in later work that attempts to influence MCF with magnetic fields, among others. These publications should be available shortly.

Another effort to compute the effect of the strong force on energy levels, lifetimes and sticking of $\mathrm{dt} \mu(00)$ and $\mathrm{dt} \mu(01)$ states with our random tempered basis sets and logarithmic boundary conditions, is being written up. It seems that with assumptions similar to those made in Ref. [5], very similar effects on these properties are found. This need not have been so, because our basis is very different, and numerical procedures used are very different. This computational capability was developed as a precursor to unfinished efforts to include strong force effects in a more sophisticated manner (see below).

\section{Incomplete Work}

For more than the past two years, we have struggled with the formulation of a more rigorous, and workable theory for the nuclear effects of (alpha) sticking in d-t fusion from 
dt $\mu$ states. Efforts with L.C. Biedenharn, A. Stahlhofen, M. Danos, J. Rafelski, B. Mueller, K. Szalewicz and all our coworkers have not produced such a theory. The problems are conceptual, mathematical and physical in nature (with different emphasis by different people). We all agree that the heuristic formula for sticking is unsatisfactory, if not uncontrollably approximate. But we could not agree on the rôle of the (11) states, the Auger transition to the (01) and (00) states to "prepare" the fusing states, nor on the proper computable treatment of the final continuum $(\alpha \mu \nu)$ states. We all feel there is something there, but we are not able to get the effect properly exposed beyond the heuristic formula. Some papers have been written by Biedenharn, Danos, Mueller and Rafelski without my coauthorship, see their final reports for details.

On the positive side, we did develop the capability to compute the variance for $\mathrm{dt} \mu$ states. These confirmed, by their smallness, the remarkable accuracy of the the Rayleigh-Ritz variational calculations performed before. This tool could be "reactivated" in later efforts to tackle the unfinished project summarized above.

\section{References}

1. "Sticking Fraction Calculations of $\mathrm{td} \mu$ and $\mathrm{dd} \mu$ Using Random Tempered Basis Sets," S.E. Haywood, S.A. Alexander and H.J. Monkhorst, Phys. Rev. A.

2. "Muon Reactivation in Muon Catalyzed d-t Fusion from Accurate p-He+ Stripping and Excitation Cross Sections," C.D. Stodden, H.J. Monkhorst and K. Szalewicz, Phys. Rev. $\mathrm{A}$, in press.

3. "Nuclear Fusion Rates of Muonic Molecular Ions," S.A. Alexander, P. Froelich and H.J. Monkhorst, Phys. Rev. A, in press.

4. "On the Accuracy of Muonic Molecular Ion Calculation," S.A. Alexander and H.J. Monkhorst, J. Phys.

5. "Effects of Nuclear Forces in Muon Catalyzed Fusion. Nonadiabatic Treatment of Energy Shifts and Fusion Rates for S States of td $\mu$," K. Szalewicz, R. Moszynski, A. Scrinzi, X. Zhao, B. Jeziorski, W. Kolos, P. Froelich, H.J. Monkhorst and A. Velenik, Phys. Rev. A.

6. "Cold Fusion Rates for Hydrogen Isotopic Molecules," K. Szalewicz, J.D. Morgan III and H.J. Monkhorst, Phys. Rev. A, 40, 2824 (1989).

7. "Simple Yet Accurate Model for Coulomb Barrier Penetration in Cold Fusion," J.D. Morgan III and H.J. Monkhorst, Phys. Rev. A (submitted).

8. "On Fusion/Fission Chain Reactions in Fleischmann-Pons 'Cold Fusion' Experiment," $S$. Anghaie, P. Froelich, and H.J. Monkhorst, J. Electroanalytical Chem. 

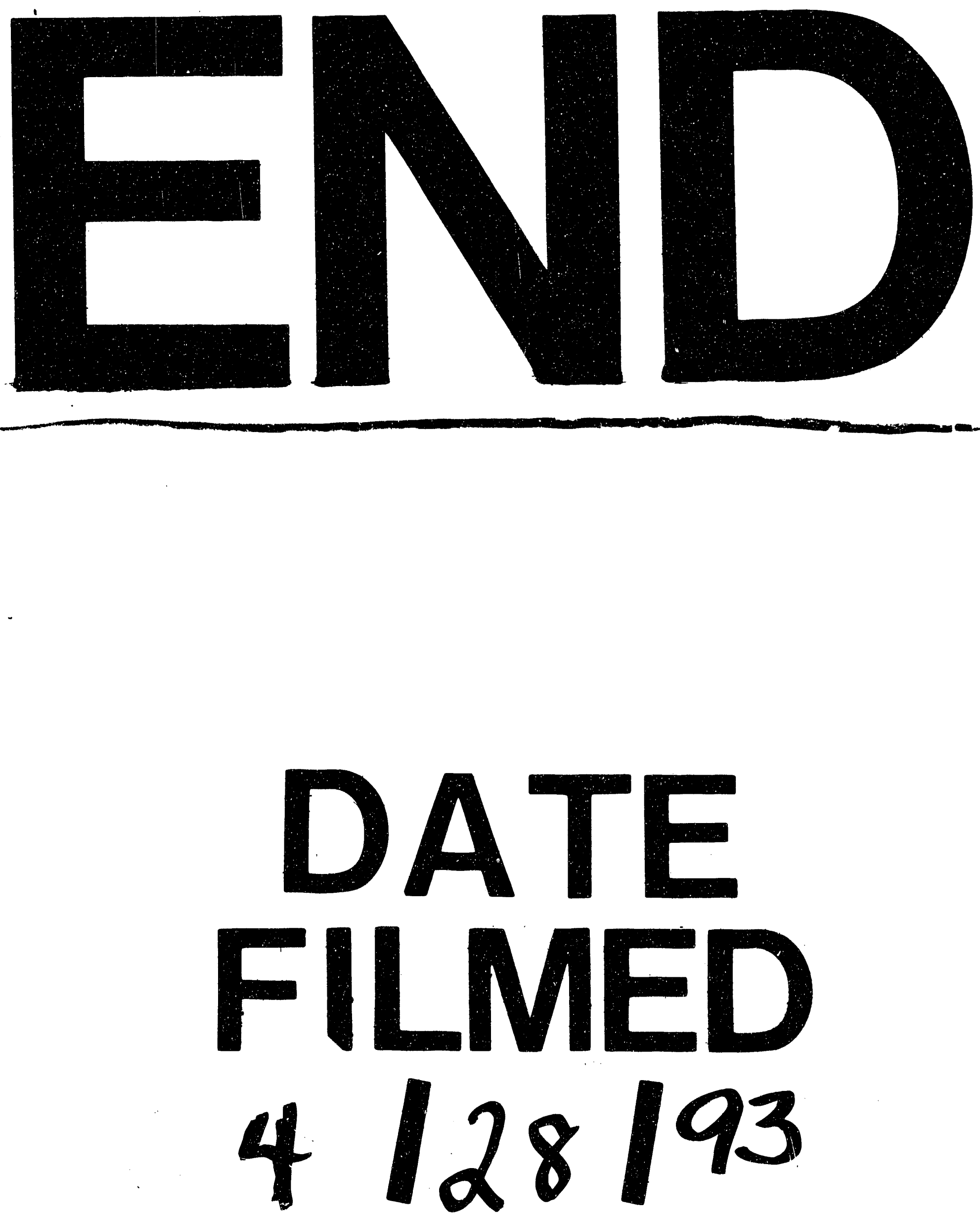
